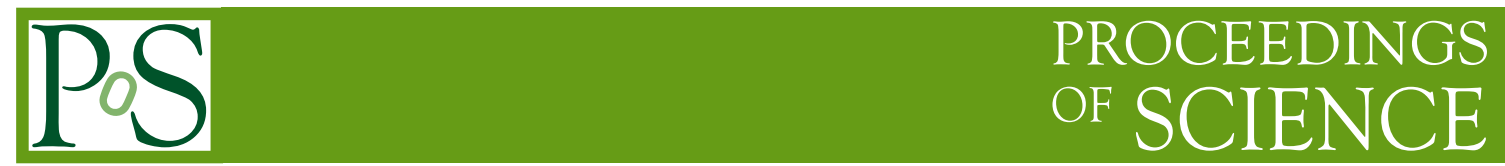

\title{
Standard Model high mass Higgs search at DZero
}

\section{Boris Tuchming*}

CEA Saclay

E-mail: tuchming@cea.fr

The data recorded by the $\mathrm{D} \emptyset$ experiment at the $1.96 \mathrm{TeV} p \bar{p}$ collider Tevatron provides the opportunity to search for the production of the Standard Model Higgs boson. With up to $6.7 \mathrm{fb}^{-1}$ of analyzed data, the search in the Higgs boson range of mass of [130 - 180] GeV shows no significant excess above the Standard Model background expectations. Thus limits are set on the Higgs boson yield as a function of its mass.

35th International Conference of High Energy Physics

July 22-28, 2010

Paris, France

* Speaker. 


\section{Introduction}

The Standard Model (SM) of particle physics is built on the existence of the electroweak symmetry breaking. In spite of the SM numerous experimental successes and constraints, the details of the symmetry breaking mechanism are still unknown. In its minimal form, this mechanism is bound with the existence of a yet to be observed single scalar particle called the SM Higgs boson. At the present time, finding the Higgs is one of the most topical goals of particle physics. Thus it is also a primary objective for the $\mathrm{D} \emptyset$ experiment which analyzes the $p \bar{p}$ collisions from the Tevatron collider, at center-of-mass energy of $1.96 \mathrm{TeV}$. As of spring $2010 \mathrm{D} \emptyset$ has recorded an integrated luminosity of $\mathscr{L} \simeq 7 \mathrm{fb}^{-1}$.

Within the SM, the mass of the Higgs boson is the only free parameter of the Higgs sector. Constraints on its value result firstly from direct searches performed on the electron-positron collider LEP (1989-2000) which conclude its mass should be heavier than $114.4 \mathrm{GeV}$ [1]. Secondly, indirect constraints are obtained when accounting for the Higgs boson's quantum effects in various electroweak measurements. They yield an upper bound of $190 \mathrm{GeV}$ at $95 \%$ Confidence Level (CL) [3].

These direct and indirect constraints define a range of mass of $110<m_{H}<200 \mathrm{GeV}$ to look for the Higgs boson, perfectly suitable for the Tevatron energy reach. Indeed, in early 2010 the Tevatron experiments, CDF and DØ, have extended the direct constraints on the Higgs boson by publishing the combined result of their searches [2]. Using up to $5.4 \mathrm{fb}^{-1}$ of data they exclude the existence of a SM Higgs boson in the mass range [163-164] GeV at 95\% CL.

This proceedings presents the status of the search by the the $\mathrm{D} \emptyset$ collaboration for Higgs bosons at high mass, namely $135<m_{H}<190 \mathrm{GeV}$, in the different channels of interest. The search for lower mass Higgs boson is described elsewhere in this proceedings [5]. All limits hereafter are given at $95 \% \mathrm{CL}$.

\section{Higgs boson production, decays and search channels}

The dominant production mode is the gluon fusion process, $g g \rightarrow H$, with a cross-section going from 1200 to $300 \mathrm{fb}$ along the range $[115-180] \mathrm{GeV}$. Further sensitivity is achieved while accounting for the associated production modes $q \bar{q} \rightarrow W H, q \bar{q} \rightarrow Z H$, and also for the vector boson fusion process, $q \bar{q} \rightarrow H q \bar{q}$, which are roughly one order of magnitude rarer than $g g \rightarrow H$.

For mass lower than $135 \mathrm{GeV}$, the Higgs boson decays predominantly into a pair of b-quarks, while for mass higher than $135 \mathrm{GeV}$, its dominant decay mode is a pair of $W$ vector bosons. This defines what is called at Tevatron the low mass and high mass ranges. In the high mass range D $\varnothing$ has searched for the Higgs production in three channels which are defined by the decay products of the $W$ 's

The di-lepton $+E_{T}$ channel corresponds to the simultaneous decays of two $W$ 's to electrons or muons (plus neutrinos). It represents a small branching ratio $(\simeq 6 \%)$ but a clean signature. The lepton+jets channel corresponds to the case when one $W$ decays to leptons while the other one decays to a pair of quarks. Its branching fraction is sizable $(\simeq 30 \%)$ but it suffers from a high SM background arising from $W+$ jets production. The third channel arises from the associated 
production of the Higgs that may yield a final state with three vector bosons (mainly three W's). This allows to search for the clean but rare signature of two leptons of same charge.

\section{Backgrounds and analyzis strategy}

A first background for these channels arise from the $W+$ jets and $Z+$ jets production. Data driven corrections are employed to model properly the recoil of the $Z$ or $W$ bosons. The second source of backgrounds comes from di-boson events. Special care must be taken for the $W W$ production since it is an irreducible background. Thus NLO correction are employed to model properly the lepton opening angle and di-boson $p_{T}$. The production of top quark pairs also yields two $W$ bosons which consitutes another type of background. The last source of background consists of QCD multi-jets production where the jets are mis-identified as leptons. This kind of instrumental background is determined in the data itself using control samples.

As electrons and muons have different instrumental backgrounds and different momentum resolutions, the analyzes are split according to the lepton flavors to achieve a better sensitivity. To further increase their sensitivity, the analyzes also make use of multivariate techniques (MVA), either boosted decision trees or artificial neural networks. They combine into a single discriminant many pieces of information that describes the event topology, the lepton kinematics, the lepton quality, the jet activity and $E_{T}^{\prime}$ related variables, that gauge whether the $E_{T}^{\prime}$ is significant or due to a mismeasured object. They are optimized for each sub-channel and each tested Higgs boson mass hypothesis.

The final MVA discriminant is employed for the statistical analyzis of the data, to probe whether the data are signal-like or background-like and to derive limits on the Higgs boson yield in the case when no signal-like excess has been observed. This analyzis accounts for the systematic uncertainties on both the signal and the backgrounds, their correlations and the way the uncertainties may modify the discriminant variables.

\section{Analyzes and results}

\subsection{Di-lepton $+E_{T}$ channel}

In this channel, the signature consists of 2 isolated high $p_{T}$ leptons and a large transverse $E_{T}$. For ICHEP 2010, the $e \mu$ channel has been updated using $6.7 \mathrm{fb}^{-1}$ of data with respect to the combined $e e+\mu \mu+e \mu$ analyzis published ealier this year and based on $5.4 \mathrm{fb}^{-1}$ [2]. This new $e \mu$ analyzis splits the final states according to their jet multiplicity $(0,1$ and more than 2 jets). Thus it achieves a better descimination against top pair events, and a better sensitivity toward $\mathrm{H}+\mathrm{jets}$ final states (their yield are actually more important for low masses). It also includes b-tagging veto that reduces further top pair backgrounds and more efficient lepton identification criteria which increase the overall acceptance by roughly $15 \%$. The final statistical analyzis of the multivariate discriminant distribution does not show excess with respect to the background expectations and limits are set.

Using $5.4 \mathrm{fb}^{-1}$ of data, the limits on the Higgs boson yield for $m_{H}=165 \mathrm{GeV}$ are $\sigma_{95} / \sigma_{S M}=$ 1.55 (1.36 expected) for $e e+e \mu+e \mu$ channels and $\sigma_{95} / \sigma_{S M}=1.99$ (1.93 expected) for $e \mu$ only [2]. The updated $e \mu$ analyzis based on $6.7 \mathrm{fb}^{-1}$ obtains a limit of $\sigma_{95} / \sigma_{S M}=1.39$ (1.62 expected), as 
seen in Fig. 1. The expected sensitivity for this channel is therefore improved by $18 \%$ twice better than what could be naively expected from the scaling with $\sqrt{\mathscr{L}}$.

\subsection{Di-lepton $+E_{T}$ with a $4^{\text {th }}$ generation of fermions}

The search for di-lepton $+Z_{T}$ final states can be re-interpreted in a straightforward fashion within frameworks going beyond the SM, such as a in models with a $4^{\text {th }}$ generation of heavy fermions. In those models the gluon-gluon-Higgs coupling is scaled by a factor of 3 with respect to the SM coupling and the $g g \rightarrow H$ process is enhanced by a factor 9. This makes both Tevatron experiments able to observed the production of the Higgs boson.

The CDF and DØ collaborations have published [4] the combination of their results for this search. They are based on the SM di-lepton $+Z_{T}$ analyzes, that have nonetheless to be slightly retuned to account for the extended mass reach which modifies the event topology and kinematics.

No excess of events have been found above the SM expectations, which allows CDF and DØ to exclude the Higgs boson masse range of $[130,210] \mathrm{GeV}$, in the $4^{\text {th }}$ generation infinite mass scenario.

\section{Lepton+jets channel}

In this channel, the signature consists of one isolated high $p_{T}$ lepton a large $E_{T}$ and two high $p_{T}$ jets. If the Higgs boson mass is higher than $160 \mathrm{GeV}$, two real $\mathrm{W}$ bosons are produced. In spite of the undetected neutrino, one can reconstruct the full event kinematics using the $W$ mass as a constraints.

Searching for the Higgs boson in this a final state is a challenge because of the overwhelming $W+2$ jets background which has to be well understood and modeled thanks to control data samples. The DØ experiment presents for the first time at ICHEP 2010 the search in this channel based on $5.4 \mathrm{fb}^{-1}$ of data. No significant excess is found in the final discriminant distributions which allows to set constraints on the SM Higgs production. For $m_{H}=165 \mathrm{GeV}$ a yield higher than $\sigma_{95} / \sigma_{S M}=3.8$ (5.5 expected) is excluded (see Fig. 1).

\subsection{Final state with two leptons of same charge}

In this channel, the signature consists of 2 isolated high $p_{T}$ leptons of same charge and a large transverse $E_{T}$. As the measurement of the lepton charge is critical in this analyzis, high quality criteria are demanded for the lepton track reconstructed by the central tracking system. The charge mis-identification rate is derived from control samples in the data.

For ICHEP 2010, a new analyzis of $5.4 \mathrm{fb}^{-1}$ has been presented, that superseds previous results based on $3.6 \mathrm{fb}^{-1}$. For each of the final states (ee, e $\mu, \mu \mu$ ) the sensitivity is enhanced by training two specific boosted decision trees (BDT). The first one is dedicated to reject the instrumental background, which is made of Drell-Yan events where one of the charge has been mismeasured, and from multi-jets events where jets yield reconstructed leptons. The second decision tree is trained against the remaining background, made of mostly $\mathrm{W}+$ jets and di-boson events.

No excess of events have been found above the SM expectations in the distribution of the second BDT, so that this analyzis set limit on the Higgs boson yield. At $m_{H}=165 \mathrm{GeV}$ a yield higher than $\sigma_{95} / \sigma_{S M}=7.2$ (7.0 expected) is excluded (see Fig. 1). This can be compared to the 

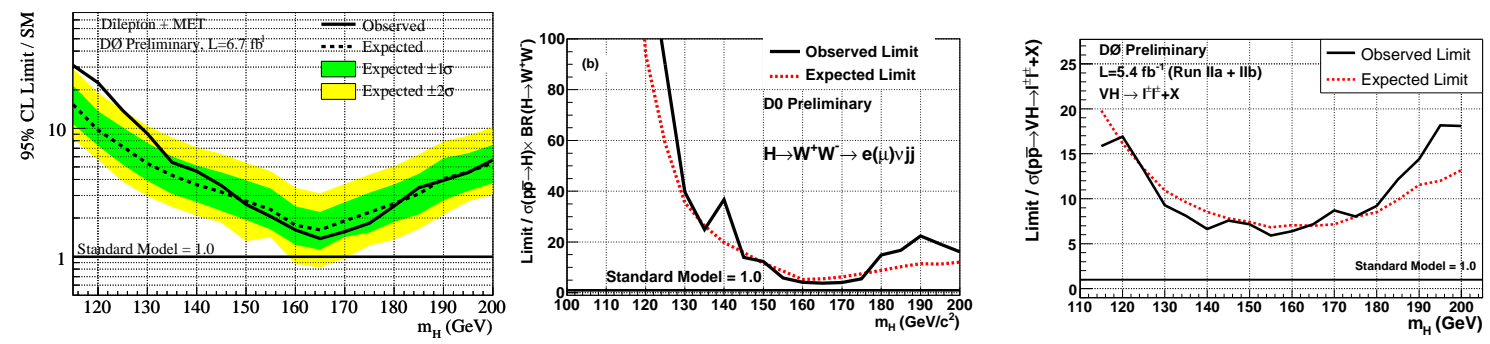

Figure 1: Limits on the Higgs boson yields obtained for the three new search results. On the left $e \mu+E_{T}$ channel, in the middle lepton+jets channel and on the right same charge di-lepton channel.

$3.6 \mathrm{fb}^{-1}$ analyzis expected limit which was $\sigma_{95} / \sigma_{S M}=10.5$. The improvement on sensitivity is therefore twice larger than the naive scaling with $\sqrt{\mathscr{L}}$.

\section{Conclusion}

DØ presented at ICHEP two updated analyzes and a new search channel that are looking the for the Higgs boson at high mass. These channels are part of the DØ Higgs combined results and the CDF-DØ combination which are both discussed elsewhere in this proceedings [6, 7].

Although the experiment alone is not yet sensitive to the production of the Higgs boson, these three new results demonstrate that the sensitivity increases faster than the square root of luminosity. If $\mathrm{D} \varnothing$ and CDF keep improving their analyzes like this, and provided that Tevatron keeps taking data up to 2014, they should be able to see evidence for the Higgs boson production over the entire mass range of $[110,190] \mathrm{GeV}$ by that time.

\section{References}

[1] R. Barate et al. [ALEPH Collaboration and DELPHI Collaboration and L3 Collaboration and OPAL Collaborations and LEP Working Group for Higgs Boson Searches], "Search for the standard model Higgs boson at LEP,” Phys. Lett. B 565, 61 (2003) [arXiv:hep-ex/0306033].

[2] T. Aaltonen et al. [CDF and D0 Collaborations], "Combination of Tevatron searches for the standard model Higgs boson in the W+W- decay mode,” Phys. Rev. Lett. 104 (2010) 061802 [arXiv:1001.4162 [hep-ex]].

[3] The Lep Collaborations and the LEP Electroweak Working group, arXiv:hep-ex/0712.0929 (2007). Update for spring 2010 at http://lepewwg.web.cern.ch/LEPEWWG

[4] T. Aaltonen et al. [CDF and D0 Collaboration], "Combined Tevatron upper limit on gg->H->W+Wand constraints on the Higgs”, Phys. Rev. D 82, (2010) 011102

[5] Y. Enari, Standard Model low mass Higgs search at DZero, this proceedings.

[6] M. Mulhearn, Combination of Standard Model and beyond Standard model Higgs searches at DZero, this proceedings.

[7] B. Kilminster, Higgs searches at the Tevatron, this proceedings. 